\title{
Sequential Card Activities of Recollect Experience for Elderly with Dementia
}

\author{
Fang Lin Chao*, Boxiu Fanjiang \\ Department of Industrial Design Chaoyang University of Technology, 436, Taiwan R.O.C
}

\author{
A R T I C L E IN F O \\ Article history: \\ Received: 11 May, 2018 \\ Accepted: 28 July, 2018 \\ Online: 10 August, 2018
}

Keywords:

Sequential card

Dementia

QR code

App.

\begin{abstract}
A B S T R A C T
In this study, the authors propose a modified design of sequential card activities to recollect experience for the elderly with Dementia. The cards and App were designed to complement each other for Nostalgia-based support. Image and text are utilized to trigger memories that bring out shared experience from the patient. By using suitable trigger elements, group sharing, and physical objects, one can enhance the memory recall. The procedure of rice dumpling preparation and making were listed in a sequential step and printed on cards. Cards are shuffled, and the elderly are asked to sort these cards to right sequence with the help of QR scanner. In multiple theme scenarios, different groups of object making steps were mixed, and the player needs to select cards that related to a specific group. Using actual items and activities, the triggered of elder's experience was confirmed. The qualitative testing results showed that the elderly enjoyed the activities. Those events verified the effectiveness of the proposed method.
\end{abstract}

\section{Introduction}

There had been an increasing trend for the number of patients with dementia in recent years, as well as a trend in which the average age for patients with dementia is becoming younger. This situation has been a challenge in the country where the amount of caregivers is low compared to the elderly population. The medical community has not found a cure for dementia; however, the condition often follows some pattern. Characteristically, foreign workers hired as caregivers do not make use of community-based services as they care for the elderly. Many efforts had been developed to support the elderly with dementia. For instance, training program, caring direction and technologies have been utilized to investigate and improve patient well-being [1-3]. The designer should realize that an integrated approach is necessary to address the cognitive, emotional and motivational aspects.

Although low-tech external memory aids have limitations, these limitations may be addressed by smart assistive technologies. Mihailidis and Fernie had concluded in a study that people with dementia assisted by context-aware computing [4]. "The research illustrates developmental training needs for dementia nursing to improve the efficacy of assessments and therefore interventions" was proposed by Carradice et al. [5]. Banerjee discuss the quality of care in dementia, co-resident spouse care with Alzheimer's dementia. Training benefits on cognitive, behavioral, and global functioning in patients with Alzheimer's disease were reported [6].

\footnotetext{
*Corresponding Author: Fang Lin CHAO, email: flin@cyut.edu.tw
}

If possible, technologies should be designed to be friendly and noninvasive.

We had observed that some patients fail to remember recent events quickly, but can recall events from the distant past. To address this, designers need to include an individual's environmental context and social relationship during their design process. Group-based nostalgia induced recollect experience may take different forms in the lives of each elderly. "During bus trip in a distant city, nostalgia was identified as a key element of the experience for socializing" [7]. In elder care center in Taiwan, some may reminisce through sing old traditional songs together, while others may look at old photos of buildings or sceneries, or read letters from the past from acquaintances. Recollect experience can be an image, text or sound track that can trigger the memory. In the instance where the caregiver and the elder have no close relationship, the activity seldom triggers any feedback. This problem indicates that the event is not sufficient, and the reaction was rendered as non-existent. As shown in Figure 1, it's difficult to communicate since they may not know much about the elder's background, so professional activities are needed.

\section{Objectives and Methods}

The goals and approaching of this study are:

1. Design sequential card activities of recollect experience that are suitable for patients with memory loss; trigger feedbacks from them using the appropriate images and text. 


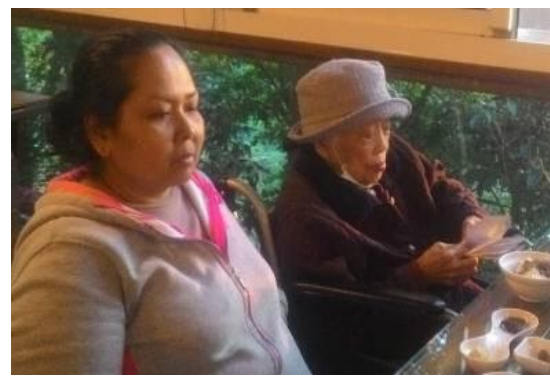

Figure 1. Relationships between person help recollect experience: A foreign helper has less in common with the elder, which causes less memory recollect .

2. To propose a group activity, through the mutual agitation among members; the seniors with dementia can discover the differences between others and arouse interest. Based on personal characteristics, actual items were utilized to trigger past experience.

An improved design was used so that participants can use it actively without technology barrier. In the nostalgia-based supporting, different presentation of information and background memory elements can enrich interaction. During this study, the designer and the elderly play with the sequential card game together. The designer can test the system, collect feedback, and implement improvements in the newer version. Sections III, IV and VI describe our evolutional development cycles from the initial concept.

\section{Arrange Sequential Card Activities}

There are memories or facts in memory backtracking. Consciousness not only contains symbols, words, or phenomena but a combination of life experiences. The feature of cards game is that each card has its facts and events. When any card wakes up the user's memory, he can continue to explore the relationship between the cards. At the time of exploration, the life experience began to be excavated. The card game proposed in the previous reference applied to the wake of the experience of the elderly [8]. This article will further elaborate on the relevant content and details.

\subsection{Design approach}

Information technology used in many ways. For example, we can implement card games within touchscreens with software; because the one operating the app needs to be familiar with the use of mobile phones, the elderly may not suitable. Although the design has the greatest flexibility, we still adopt "supportive" proposals. The APP is only a supporting character. If there is any doubt, one can use a scanner to get help. It is as though we are asking others a question. Because the sequential card games are with a physical object that can be touched, it is suitable to link with the life experience of the elderly and reduces the fear.

\subsection{Concept realization}

Software is used as a means to reduce the stress in taking care of elderly with dementia; since they often repetitively ask the same questions, the average caretaker may be irritated from answering the same questions over and over again. Thus, the app is advantageous in that it can make a reply anytime, and answer related questions immediately, and won't be irritated from repeating the same answers.
Table 1. The concept shifts concerning the user confidence, where fluency means "the possibility of without obstacles during the game."

\begin{tabular}{|l|c|c|}
\hline & $\begin{array}{l}\text { standalone } \\
\text { within screen }\end{array}$ & $\begin{array}{l}\text { supportive } \\
\text { explanation }\end{array}$ \\
\hline flexible & +++ & -- \\
\hline fluency & + & ++ \\
\hline affordance & --- & +++ \\
\hline
\end{tabular}

Based on Table 1, we utilize app as a supporter or explainer. Sequential card was designed (Figure 2) with the theme of wrapping rice dumplings (zongzi). For each step card, a proper image and description text should be matched. The cards are shuffled in random order before the game, and the elderly are asked to sort these cards into the correct sequence.

As technology is added in design, we have to consider whether the senior will reject or not feel accommodated to using these tools. Thus, in the card design, pictures or text is still shown on the front side, while the tag placed on the back side. The user won't often be distracted by tag. When the user wishes to seek help, they can get pertinent information by moving the card to a fixed position. QR code was placed on the back of each card. While the elderly see the image, the scanner read the QR code and activate necessary audio explanation with adjustable volume and speed (Figure 3, 4). During the process, if the elderly ever want to express anything about personal experience, question or feedback, the elder's voice response would be collected.

Remaking the step cards would be inconvenient when there are plenty of triggering elements. A reconfigurable card holder can be used to different sequential cards. Considering game's flexibility, while selecting the proper subject, the card's corresponding QR code [8] will not require re-setting.

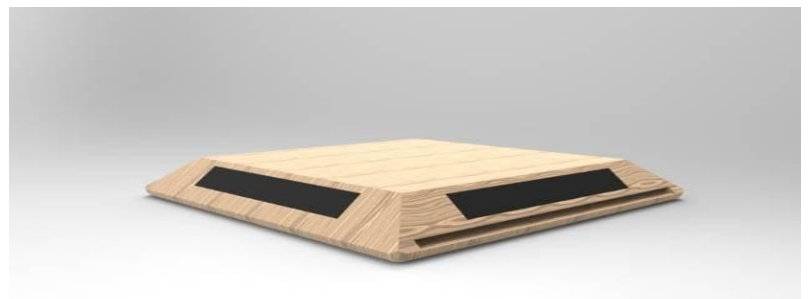

Figure 2. Continuous applicable card holder. A reconfigurable slot is placed on the side wall for inserting a card. A user can change the theme more efficiently with this design.

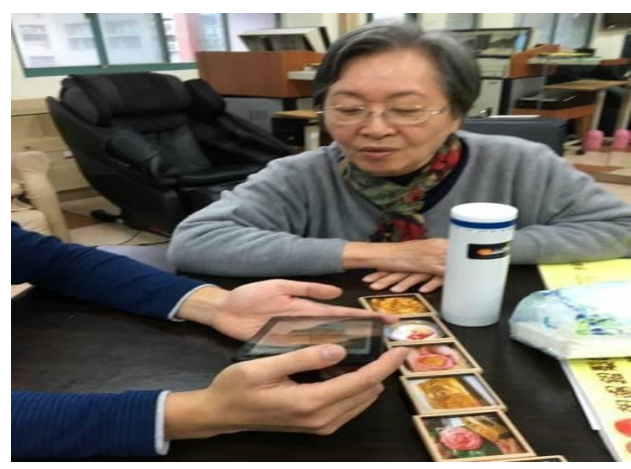

Figure 3. Scanning will take some time for elderly. 


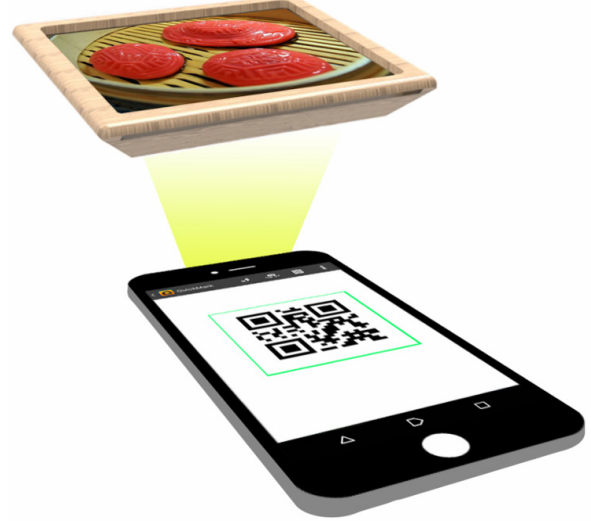

Figure 4. QR code placed at the back of the sequential card. With an unstable hand, the user need moves the card to the designated position stably to scan the code.

During the trial, we noticed a few unstable hands during code scanning. In the modified design, QR code is placed at the back of the card to make it more convenient for the elderly. When the user can't hold the phone steadily with their hands, scanning will take some time. We can make the response time from scanning faster by placing the phone in a fixed position (Figure 5). If the user can hold the phone steadily and successfully operate the app, then a fixture for scanning won't be necessary.

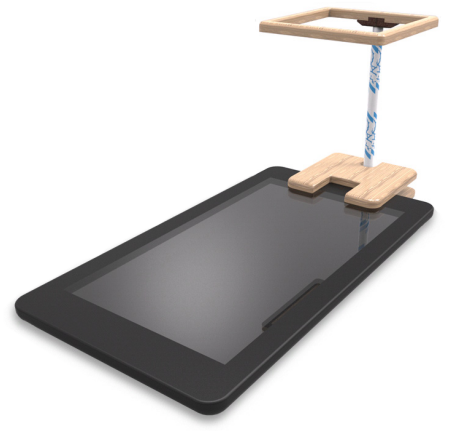

Figure 5. When the user can't hold the phone steadily with their hands, the supporting fixture make the response time from scanning faster

\section{Mobile App Design}

The app was made using MIT App Inventor 2 which use module block in programming. App design arranged procedure screens as Figure 6. App Inventor programs blocks (Figure 7) represent sensing the measured value through the QR connection.
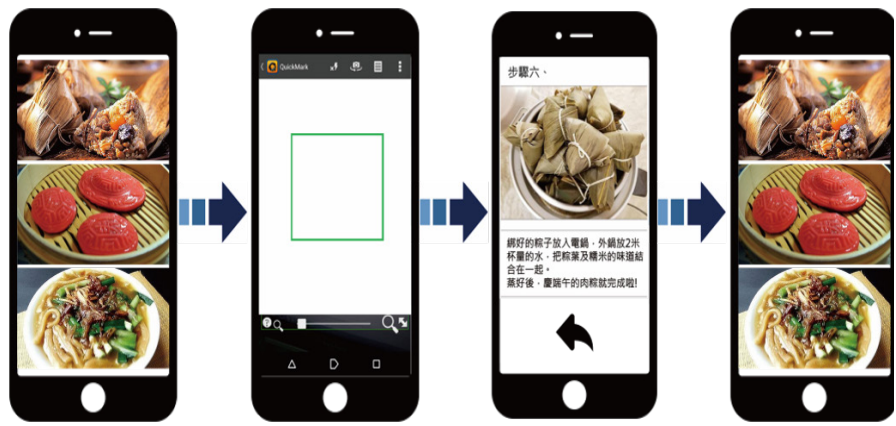

Figure 6. When the user clicks on an image, a scanner pops up. As the user scans the corresponding image on the card, texts detailing each step of the food preparation process pops up.
Students in the industrial design department seldom use programs, so they often forget the syntax and structure of the program. The module block can connected to another neighborhood block. It will be pushed apart automatically if it is not appropriate. Therefore, the designers pay attention to the main logical relationship between the program block and the selection of the module. The connection between Bluetooth blocks, we need to setup the paired connection and send the appropriate data to the other party. As shown in Figure 7, the program block and the link of the modules is quite simple; although the blocks are simple, they still achieve powerful functions.

An event is an action that takes place. App Inventor II uses event handler blocks to specify how the program responds to individual circumstances. There is a user-initiated and automatic event. Every time the interval is up, a timer event triggered. Buildin sensors also initiated events when the user made a specific movement or orientation.
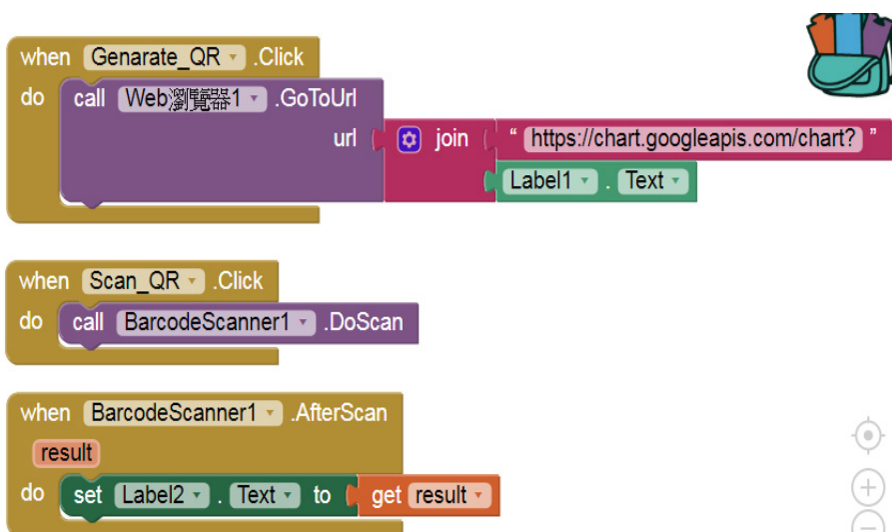

Figure 7. QR scan programming with modulated App Inventor blocks

\section{Multiple theme Scenarios}

The more advanced games are organized for higher-skilled people; three recollect experience activities are available with different series of cards. Six step card blocks are cut to be in a 45 degree plane. By touching the magnets on each side, we can assemble six side blocks into a cube (Figure 8).

In multiple theme scenarios, different object making process are mixed, the player needs to select cards that related to specific objectives. After grouping those cards, one need to arrange those cards in proper sequence. When the blocks are organized into a cube, there is more variation for the player. The player cannot see all figures at once. The elderly need rotate the cube, and think about the category relationship between the images, and try to exercise their memorization ability more.

Assuming three recollect experience activities are available (making of rice dumpling, red turtle cake, and rice noodle soup), and each of these three groups has 6 step cards to be reordered. Put together cards of the same order, and make six cubes, and give those to the user. The researcher provides a questionnaire to the player. For example, if a user chose a turtle cake theme, the user has to make the related sides of each cube face upwards and rearrange these cubes. 


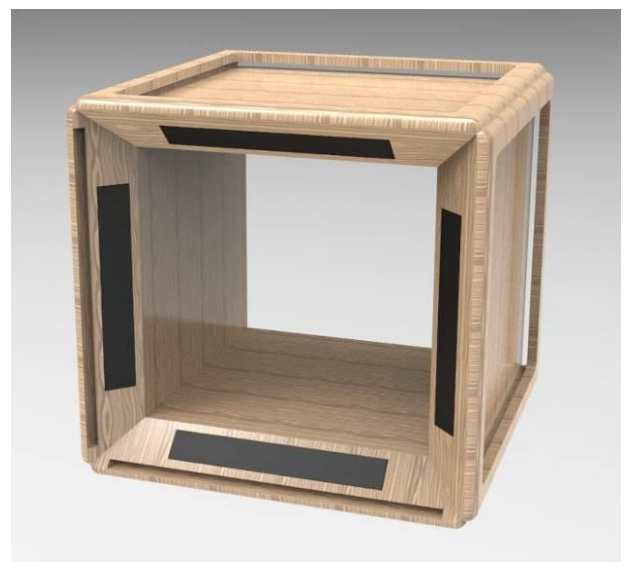

(a)

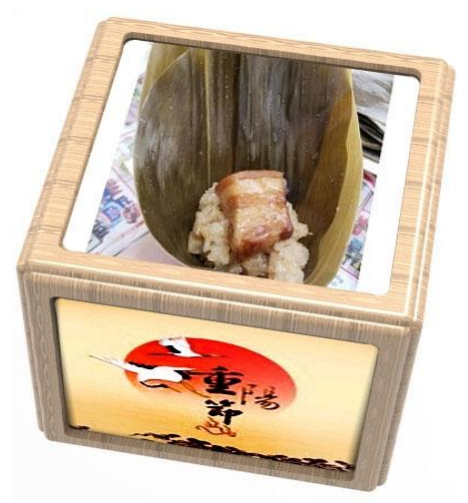

(b)

Figure 8. Three-dimensional sequential card game, (a) magnets on the edge of a card allows each card to be attached to the cube, (b) the combined cube. In multiple theme scenarios, each card belongs to a different theme.

The flow chart for the sequential card game indicated in Figure 9. The explanations of each card are stored at separate lists. Player need recall experience about the steps of trigger elements and the timing relationship between cards. The cards help people focus and trigger the memory of a player.

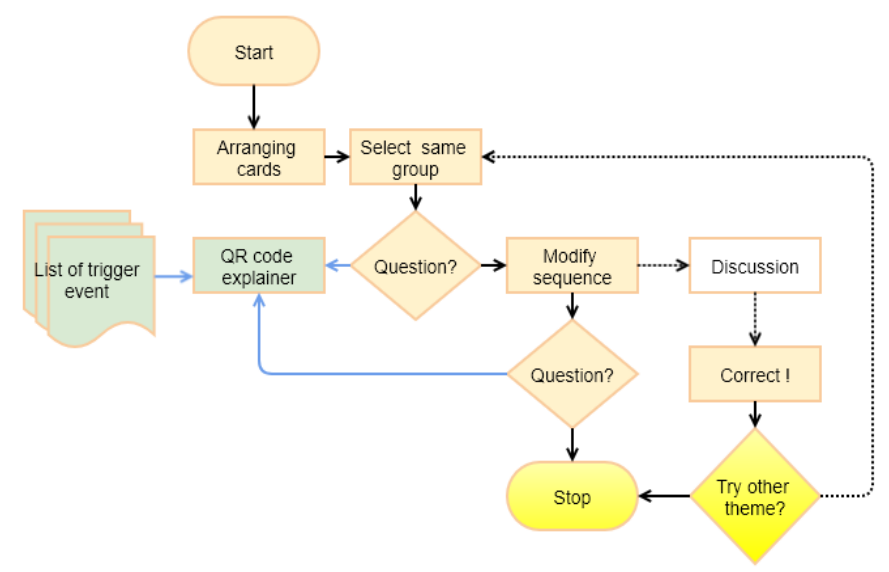

Figure 9. Sequential card and app flow chart indicates recollect experience with the support of QR code explainer. Question and discussion may be triggered while modifying sequence. After finish the sequential placement, users can try another theme.

\section{User Testing}

\subsection{Elderly center interview}

During the evaluation process, the participants actively talked to us about their experiences in making rice dumplings. For example, one mentioned a tip on how to make tastier dumplings, and one made a suggestion based on how her mother made it. The two testers shared the experiences with each other, which enhanced the recollect experience treatment's effect. The lady (in Figure 10) talked about when she was little, her family made red turtle cake during Christmas time, because her family treats Christmas like Lunar New Year, and this was their way of celebration. Tester $\mathrm{C}$ came from mainland China. She is a retired teacher. She is quiet and not very talkative to others. She likes to cook at home; she usually eats noodles and knows how to wrap rice dumplings herself. She prefers plain or slightly sweet rice dumplings with red bean paste or red dates added. Tester D is also a quiet lady with dementia. She often repeats the same thing over and over. She has more of a monotonous lifestyle and regularly watches TV. She likes to cook at home; usually, eat rice and prefer meat dumplings.

During the evaluation process, participants had made feedback about the using the sequential card. The shared message was recorded by a researcher. A five points Likert scale survey about playing the game were collected, the averaged of the score are shown in Table 2 . The single theme achieved better manipulation experience, less skill and explanations were requested. When four people play a single theme sequential card game together, the games are fluent and emotional engagement (talkative to others) are high (score above the average value). This activities indicated positive feedback. They are grateful to share individual experiences. The communication links those elderlies together, and make nostalgia-based treatment more effective.

The multiple theme achieved less manipulation experience, more skill and memory function are request for proceed the game. More explanations were requested. Arranging magnets placed at sides and organized into a cube is a challenge for elderly with less detail manipulation skill. We suggested multiple theme is suitable only for advanced users.

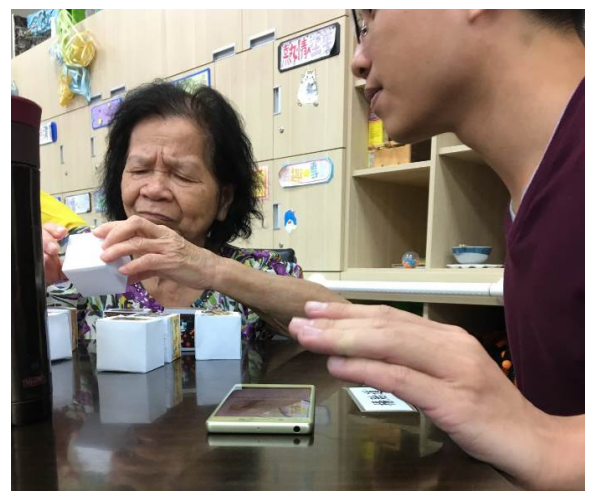

Figure 10. User observation of multiple theme, to provide proper image with good resolution and teach user to get explanations through the support of QR code.

\subsection{Follow-up personal activity}

Through group activities, the mutual interaction among members, old people with dementia discovered the differences of others. Then, a follow-up private action based on actual items and 
activities was conducted to confirm when measurable memory is triggered.

Table 2. averaged feedback about using the product (5 points Likert scale)

\begin{tabular}{|l|c|c|}
\hline & single theme & multiple themes \\
\hline manipulation skill & 4.25 & 3.25 \\
\hline request explanation & 3.00 & 4.50 \\
\hline fluent of game & 4.25 & 3.00 \\
\hline emotional engage & 4.25 & 3.25 \\
\hline talkative to others & 3.75 & 3.00 \\
\hline
\end{tabular}

Our participant is a 90-year-old woman with dementia; she often repeats the same thing and does not notice it. She has a monotonous lifestyle, and usually watches TV, and sleeps for longer durations. Based on past life experience, the selected personal activity is rice dumpling making. We prepared objects such as bamboo leaves, strings, and red dates (Figure 11).

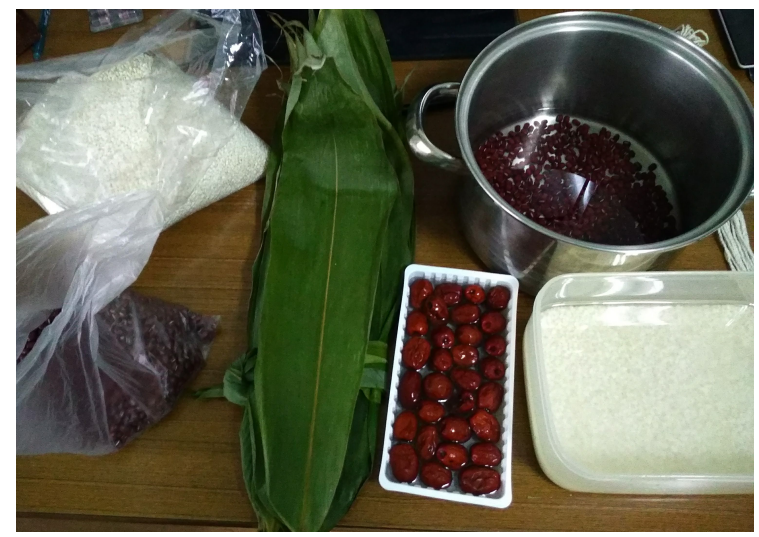

Figure 11. The prepared objects and items during personal practice and interview.

Through the initial guidance, the participant gradually recall her past experiences with rice dumpling making. We then made recordings of her descriptions with different aspect of experiences (Figure 12).

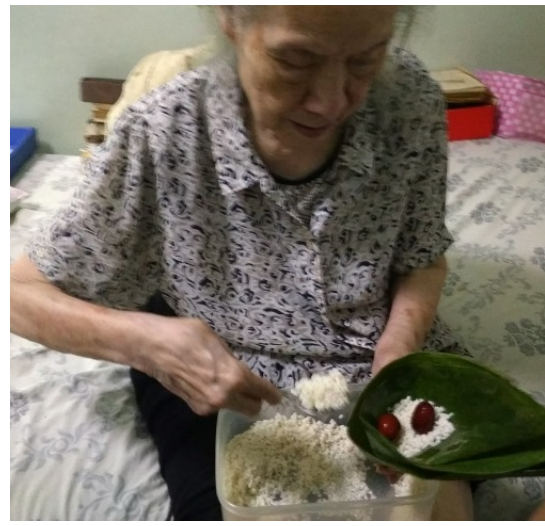

Figure 12. Personal practice and interview

Second, we will analyze the sentences (underline) in her descriptions, and categorize them into facts, estimations based on events, phenomena that may result from situations, and personal emotions. The subject's narrative was classified, and her depth of participation is presented in Table 3. During 3 hours of communication, we collect 24 minutes of voice records which are summarized below. Some paragraph was repeated to show her traits.

1. How many kilograms of rice do you have to buy? Now you can only get one serving. You have to buy a kilo of rice and soak the rice in water. Bamboo leaves must be soaked before they become soft.

2. Do you know how to wrap a rice dumpling? I thought these [strings] were worms! We put red beans with sugar, like cooking porridge. Rice must wash first. A cup of rice is fine. The rice must wash for preparing quickly.

3. Bamboo leaves must first be soaked water to be softened, which is not easy to be torn. Just put a few red dates on it. Wash them first and soak them in water, and throw a few into the rice dumplings. It will swell as soon as it cooked, and it will be delicious.

4. What is the use of dates? Red dates are used for eating ah! The boiled berry swell while cooking. There are hard shells inside the red dates, and your teeth may hurt if you accidentally bite on them. The price of bamboo leaves is high. Someone in the market sells USD, and another sells only \$2. Larger leaves are more expensive because fewer people are selling them.

5. When there is no bamboo leaf, how should you pack the rice dumplings? Smaller leaves can be used, a few pieces together. If you don't break it, you can dry it and re-use it. Bamboo leaves have a scent! Maybe someone thinks it's smelly odor.

6. How do you buy the leaf? I guess. Do you mean to purchase bamboo leaves? Would you put pork or salted eggs in dumplings? We usually eat sweet rice dumplings. Let's start wrapping! I dumped the water and, here is the material for you, bamboo leaves and rice, and strings.

7. Why is water dripping out from the rice dumplings? There is water flowing out, and a hole on the side of the rice dumplings and rice inside may come out. Why do you use two leaves to wrap it? Because a piece of a leaf will quickly be torn with force and with two pieces, it will be firm.

8. How do you tie the rope while bundling? Hold it with one hand and use the other to help. This one was too flat and not wrapped well. Someone will use teeth to help bite the string and tie it so that it's tighter.

Owing to the capacity disorder of poor hearing, the participant would often say "what you are saying I can't hear". Sometimes she asks, "Where do you come from," "Where do you go to work?" and similar typical questions. This is the typical response of dementia patients.

The details response of those activities contains logical relations and inferential information, which are different from her usual behavior. In addition to the characteristics of the sentence analysis of her recorded response, there is also the presentation of actions. For instance, each of the steps of wrapping a rice dumpling is hidden as procedural memory in the cerebellum. When the hand touches these objects, such as materials for wrapping a rice dumpling, those memories are evoked; and the previous situations can emerge. 
Table 3 . The summary of voice recording and categorizing into facts, estimations, inductions and emotions.

\begin{tabular}{|c|c|c|c|}
\hline facts & estimation & induction & emotion \\
\hline $\begin{array}{l}\text { hard shells } \\
\text { inside } \\
\text { fewer people } \\
\text { are selling } \\
\text { soak the rice } \\
\text { red dates with } \\
\text { sugar, } \\
\text { wash first } \\
\text { usually eat } \\
\text { sweet } \\
\text { water flowing } \\
\text { out }\end{array}$ & $\begin{array}{l}\text { hard shells inside } \\
\text { only get one } \\
\text { serving. } \\
\text { buy a kilo of rice } \\
\text { a cup of rice } \\
\text { rice inside may run } \\
\text { out } \\
\text { will be firm } \\
\text { two leaves to wrap }\end{array}$ & $\begin{array}{l}\text { become soft } \\
\text { will swell } \\
\text { will be delicious } \\
\text { cooked quickly } \\
\text { Small pieces } \\
\text { dry it and re-use it } \\
\text { use teeth to help } \\
\text { have a scent }\end{array}$ & $\begin{array}{l}\text { I thought } \\
\text { these were } \\
\text { worms! } \\
\text { Red dates are } \\
\text { used for } \\
\text { eating ah! } \\
\text { I guess. } \\
\text { Do you mean } \\
\text { to buy } \\
\text { bamboo } \\
\text { leaves } \\
\text { someone } \\
\text { thinks it's } \\
\text { smelly odor }\end{array}$ \\
\hline
\end{tabular}

The academic reference [9] indicates "Imagery and motion were found to share common cerebellar circuitry;" "which reflect a higher demand for the cerebellum as compared to a motion." The activation pattern observed during the execution of the finger-tothumb movement (motor preparation and timing) is in agreement with theories [9]. "Specific localizations of right superior cerebellar activation, the reaction times were significantly increased for the verbal working memory and motor control tasks" were reported [10]. From the academic reference and user observation, the presentation of actions is another crucial trigger element. It may conduct in the future design of sequential card game.

\section{Conclusion}

The main contributions in the paper are an implementation of the sequential card activities with different themes to recollect experience. The steps for rice dumpling preparation and making were printed on cards. The elderly are asked to sort these cards to right sequence with the help of a $\mathrm{QR}$ scanner. The qualitative testing results showed that the elderly enjoyed the activities. Through the game and trigger elements, non-family members or caregivers have more social interaction and involvement in personal experiences. The communication appeared among members during the group activity. The old people with dementia discovered their differences and enabled passing experiences between each other.

Based on individual characteristics, we also conducted actual items and physical activities; the participant is triggered to find out his experience that has been identified. Concerning unique features of dementia, understanding of the activation made by the elderly was confirmed. In future, the app will extend with remote communication ability to share with the friends. The presentation of motion actions is another crucial trigger element. It will conduct in future card game design.

\section{References}

[1] M.S. Bourgeois, C. Camp, M. Rose, B. White, M. Malone, J. Carr, and M. Rovine, "A comparison of training strategies to enhance use of external aids by persons with dementia," J. Commun. Disorders, 36, 361-378, 2003. https://doi.org/10.1016/S0021-9924(03)00051-0

[2] L. Clare, B.A. Wilson, G. Carter, and J.R. Hodges, "Cognitive rehabilitation as a component of early intervention in Alzheimer's disease: A single case study," Aging Mental Health, 7, 15-21, 2003. https://doi.org/10.1080/1360786021000045854

[3] S. Josefsson, L. Backman, L. Borrel, B. Bernspang, L. Nygard, and L. Ronnberg, "Supporting everyday activities in dementia: an intervention study," Int. J. Geriatric Psychiatry, 8, 395-400, 1993. https://doi.org/10.1002/gps.930080505

[4] A. Mihaildis and G. Fernie, "Context-aware assistive devices for older adults with dementia," Gerontechnology, 2, 173-189, 2002. https://doi=10.1.1.588.447\&rep=rep1\&type=pdf

[5] Carradice, Angela, Marie Claire Shankland, and Nigel Beail. "A qualitative study of the theoretical models used by UK mental health nurses to guide their assessments with family caregivers of people with dementia." International Journal of Nursing Studies 39.1 (2002): 17-26. https://doi.org/10.1016/S0020-7489(01)00008-6

[6] Banerjee, Sube, et al. "Improving the quality of care for mild to moderate dementia: an evaluation of the Croydon Memory Service Model." International Journal of Geriatric Psychiatry: A journal of the psychiatry of late life and allied sciences 22.8 (2007): 782-788.

[7] S. Fairley "In search of relived social experience: group-based nostalgia sport tourism," Journal of Sport Management, 17(3), 284-304, 2003. https://doi.org/10.1123/jsm.17.3.284

[8] Chao, Fang-Lin, et al. "Design Jigsaw puzzle and app for Recollect experience support on elderly with Dementia." Awareness Science and Technology (iCAST), 2017 IEEE 8th International Conference on. IEEE, 2017. DOI: 10.1109/ICAwST.2017.8256464

[9] Luft, A.R., Skalej, M., Stefanou, A., Klose, U. and Voigt, K., 1998. Comparing motion - and imagery - related activation in the human cerebellum: A functional MRI study. Human brain mapping, 6(2), pp.105113. https://doi.org/10.1002/(SICI)1097-0193(1998)6:2<105::AIDHBM3 $>3.0 . \mathrm{CO} ; 2-7$.

[10] Desmond, John E., SH Annabel Chen, and Perry B. Shieh. "Cerebellar transcranial magnetic stimulation impairs verbal working memory." Annals of Neurology: Official Journal of the American Neurological Association and the Child Neurology Society 58, no. 4 (2005): 553-560. https://doi.org/10.1002/ana.20604 